\title{
Stroke Pentagon: Stroke Management Approach in Resource Poor Settings
}

\author{
Chukwuemeka O. EZE ${ }^{1}$ \\ ${ }^{1}$ Internal Medicine Department, Alex Ekwueme Federal University Teaching Hospital, Abakaliki (AEFUTHA), \\ Ebonyi State, Nigeria \\ Correspondence: Dr. Chukwuemeka O. EZE, Internal Medicine Department, Alex Ekwueme Federal University \\ Teaching Hospital, Abakaliki (AEFUTHA), Ebonyi State, Nigeria. Tel: 234-703-343-2117.
}

Received: September 24, 2020 Accepted: November 11, 2020 Online Published: November 26, 2020

doi:10.5539/gjhs.v13n1p57

URL: https://doi.org/10.5539/gjhs.v13n1p57

\begin{abstract}
Stroke is a neurological condition that is characterized by sudden onset focal neurological deficit due to spontaneous cerebral vascular occlusion or rupture. It is a neurological emergency and its prevalence is very high, especially in developing countries where it assumes an epidemic proportion. It is globally the second most common cause of death after ischaemic heart disease. The poor indices in developing countries are multifactorial and related to late case presentation, ignorance, poverty, and unavailability of comprehensive and well-coordinated stroke care. There is a need to identify the available and cheap stroke management steps in the developing countries and strengthen the system to maximize the benefits in reduction of the morbidity and mortality of stroke. It is against this background that we identified Stroke prevention, acute stroke management, Stroke rehabilitation, Stroke research, and Stroke support as five pillars (stroke pentagon) in stroke management in developing countries. There is a need to sensitize the stakeholders in stroke management as highlighted in the stroke pentagon to assume more responsibility. Moreover, there is the need to have a more coordinated and concerted stroke management approach which will involve all the identified five pillars to ensure improved stroke indices in the developing countries.
\end{abstract}

Keywords: approach, developing countries, management, pentagon, stroke

\section{Introduction}

Stroke is characterized as a neurological deficit attributed to an acute focal injury of the central nervous system (CNS) by a vascular cause, including cerebral infarction, intracerebral hemorrhage (ICH), and subarachnoid hemorrhage (SAH) (Sacco et al., 2013). It is a neurological emergency and its prevalence is high especially in developing countries where it assumes an epidemic proportion. It is globally the second most common cause of death after ischaemic heart disease according to the Global Burden of Disease Study 2015 (2017), and it is projected to remain so by 2030 (WHO, 2013). It has a global prevalence of 1300.6 per 100,000 in 2017 (Avan et al., 2019).

In Africa and other developing countries, the prevalence ranged from 963 to 1,460/100,000 (Khedr et al., 2014; Ezejimofor, 2017). In Abakaliki, Nigeria, a community-based survey revealed a stroke prevalence rate of 2,700 per 100,000 amongst adult population (Eze, Kalu, \& Nnaji, 2020). It is a disease of the middle-aged and elderly population with male preponderance, though young people are not exempted (Nedeltchev et al., 2005; Varona, Guerra, Bermejo, Molina, \& de la Camara, 2007; George, Tong, Kuklina, \& Labarthe, 2011; Eze, Kalu, \& Isiguzo, 2019). It constituted $14 \%-16 \%$ of admissions in the medical wards in Nigeria (Eze et al., 2013; Ezeala-Adikaibe et al., 2014).

Ischemic stroke occurs when a cerebral blood vessel is occluded by either a thrombus or an embolus leading to focal brain ischemia. The ischemic area has a central core where brain damage is irreversible and a peripheral penumbra where there is loss of function but not irreversibly damaged. The neurological function loss is that of the ischemic core and the penumbra. The acute management of stroke aims to restore the functions of the penumbra by ensuring adequate blood perfusion. Further recovery of neurological functions during subacute and chronic stages is achieved through brain plasticity (Kolb, 1995).

Conversely, hemorrhagic stroke occurs when a cerebral blood vessel ruptures due to severe hypertension or 
defects in vascular wall integrity. This leads to the formation of hematoma within the brain parenchyma, subarachnoid space, or the ventricles. The clinical features are due to rise in intracranial pressure, meningeal irritation, and focal brain compression and ischemia. Reduction of intracranial pressure is pivotal in the management of hemorrhagic stroke.

The clinical presentation varies and depends on many factors which include the type of stroke, severity of stroke, part of the brain affected, and presence of co-morbidities. The common clinical features include hemiparesis, hemifacial paresis, speech impairment, headache, alteration in consciousness, and seizures.

The common risk factors are hypertension, diabetes mellitus, smoking, heart disease, dyslipidemia, obesity, alcoholism, and advanced age. The case fatality rate is still very high, especially in the developing countries (Walker et al., 2011; Eze, Okoro, Nwobodo, Nnaji, \& Isiguzo, 2020). In Abakaliki, Nigeria, stroke constituted $19 \%$ of deaths in medical wards (Eze et al., 2020), 24\% of deaths in medical emergency room (Eze \& Kalu, 2019), and 31\% in intensive care unit (Eze et al., 2020). The poor indices are multifactorial ranging from late case presentation, ignorance, poverty, and unavailability of coordinated and comprehensive stroke care. Hyperacute stroke interventional services like thrombolysis, thrombectomy, and embolectomy are not available in most stroke centres in Africa and other developing countries. There is a need to identify the available and cheap stroke management steps in these resource poor settings and strengthen the system to maximize the benefits in reduction of the morbidity, and mortality of stroke. It is against this background that we identified Stroke pentagon which includes Stroke prevention, acute stroke management, Stroke rehabilitation, Stroke research, and Stroke support as the pillars of stroke management approach in resource poor settings.

\section{The Stroke Pentagon}

Stroke pentagon is the five key pillars or steps in stroke management that are potentially available in the developing countries and in resource poor settings. They are cheap and available but may not be utilized fully if not highlighted. They can be strengthened and maximized to reduce the morbidity and mortality of stroke. The components of the pentagon include:

1). Stroke prevention.

2). Acute stroke management.

3). Stroke rehabilitation.

4). Stroke research.

5). Stroke support.

\subsection{Stroke Prevention}

Stroke prevention involves all the efforts put in place to ensure that people do not develop stroke. It includes health promotion and prevention, reduction, or elimination of cardiovascular risk factors. This is essential because "prevention is better than cure". Stroke prevention is the most important pillar in stroke management because it is cheap, targets the populace, and all health professionals and community leaders are involved. The target is to reduce the burden of stroke. It includes primary and secondary prevention.

Primary prevention involves prevention of stroke in people that have not had stroke, while secondary prevention involves prevention of stroke recurrence in stroke survivors. The risk factors are sought for especially amongst middle-aged and elderly individuals and also in young people with family history of cardiovascular disease. The risk factors are classified into modifiable and non-modifiable risk factors. Modifiable risk factors include hypertension, diabetes, dyslipidemia, smoking, alcohol abuse, heart disease, obesity, sepsis, and inflammatory conditions (Romero, Morris, \& Pikula, 2008), while non-modifiable risk factors include age, sex, race, and previous stroke. The emphasis is on the modifiable risk factors because they are potentially reversible. Hypertension is the most important stroke risk factor (Lackland et al., 2014).

Measures are put in place to reduce or eliminate the risk factors. The following interventional measures can be done to ensure reduction and elimination of the identified modifiable risk factors;

1). Regular physical exercise.

2). Consumption of fruits and green leafy vegetables (Owolabi et al, 2018).

3). Reduction in consumption of alcoholic beverages.

4). Cessation of cigarette smoking.

5). Reduction of consumption of refined food. 
6). Regular medical checkup.

Adherent on medications for hypertension, diabetes, dyslipidemia, and heart disease.

\subsection{Acute Stroke Management}

Stroke is classified based on time from onset into hyperacute (24 hours), acute (1-7 days), subacute (8 days-6 months), and chronic (> 6 months).

Hyperacute stroke management services like thrombolysis, thrombectomy, and embolectomy are largely unavailable in most developing countries due to high cost and lack of expertise.

Acute stroke management involves measures put in place to ensure recovery of neurological functions, prevention of another event, prevention, and treatment of acute complications during the acute phase of stroke. This is commenced immediately the patient is brought to the emergency room. Delay in commencement of acute stroke treatment may be experienced at the home, on transit and at the hospital. Acute management may extend beyond the first seven days depending on the clinical situation. Conversely, it may last for less than 7 days in a mild stroke. The patients are triaged and managed in the general medical/stroke ward or intensive care unit (ICU) depending on the severity. The acute management should be strengthened to reduce the morbidity and mortality of stroke. The patients should be managed by a dedicated stroke team coordinated by a neurologist.

Indications for admission into the ICU include;

1). Severe hypertension/hypotension.

2). Status generalized seizure.

3). Oxygen desaturation.

4). Severe alteration in consciousness (GCS $<9)$.

Management protocols for ischaemic stroke include

1). Intravenous Isotonic fluid (normal saline, ringers lactate) $2000 \mathrm{ml}$ daily (cautious fluid administration in hypertensive emergencies and in elderly patients).

2). Antiplatelets like Clopidogrel or Aspirin $75 \mathrm{mg}$ daily.

3). Maintain permissive hypertension to ensure adequate blood flow to the penumbra (MABP-110-150 mmHg, SBP-160-220 mmHg, DBP- 90-120 mmHg).

4). Reduce the blood pressure towards normal if the patient has features of hypertensive emergency or chronic end-organ failure.

Management protocol for hemorrhagic stroke

1). Intravenous $20 \%$ mannitol $300 \mathrm{ml}$ over 10 minutes, then $150 \mathrm{ml}$ over 5 minutes 6hourly for 3 days.

2). Intravenous Furusemide $20 \mathrm{mg} 12$ hourly for 7 days.

3). Reduce the blood pressure (MABP- $<120 \mathrm{mmHg}, \mathrm{SBP}-<160 \mathrm{mmHg}$ ).

4). Analgesic for headache and neck pains.

5). Stool softener and indwelling urethral catheter to avoid straining.

6). Prophylactic antiseizure medications.

General management protocol

1). Gentle graded physiotherapy.

2). Use of water or air bed to minimize risk of pressure sores.

After the acute phase, the BP will be gradually reduced to normal values.

\subsection{Stroke Rehabilitation}

Stroke rehabilitation involves the pharmacological, and non-pharmacological measures aimed at encouraging recovery of functions following stroke or optimization of the functions. These include;

1). Physical rehabilitation/Physiotherapy.

2). Swallowing rehabilitation.

3). Speech rehabilitation. 


\section{4). Psychological rehabilitation.}

5). Occupational rehabilitation.

These activities are carried out by the neurologist, physiotherapist, psychologist, speech therapist, occupational therapist, and the dietitian. Rehabilitation is usually commenced during the acute phase in the ward, though in a gentle and graded manner as vigorous physical therapy may precipitate a repeat stroke. During the Subacute and chronic stroke phases, physiotherapy is usually done on outpatient basis at the physiotherapy department or home visit.

a). Physical rehabilitation: There is strong evidence that exercise after stroke can improve cardiovascular fitness (Pang, Eng, Dawson, \& Gylfadottir, 2006),walking ability (Veerbeek, Koolstra, Ket, van Wegen, \& Kwakkel, 2011), and upper extremity muscle strength (Harris \& Eng, 2010). Physical activity goals and exercise prescription for stroke survivors should be customized for the individual to maximize compliance and long-term adherence. It should emphasize low to moderate intensity aerobic activity, muscle-strengthening activity, and reduction of sedentary behavior. The interventions include proper positioning, movement on the bed, sitting, standing, and ambulation. The above activities are focused, graded, and customized. Physiotherapists carry out the above services and it is available in most tertiary health facilities in developing countries.

b). Swallowing rehabilitation: About 51-55\% of patients develop swallowing impairment revealed by clinical testing after stroke (Mann, Hankey, \& Cameron, 1999; Smithard et al., 1996). Swallowing tests are usually done before commencement of oral feeding following stroke. Nasogastric (NG) tube feeding is used for those that have swallowing impairment. Fluid diet is given through the NG tube initially at a low volume and gradually increased as tolerated. Gastric prokinetic agents and proton pump inhibitors are used to reduce the risk of aspiration. Percutaneous endoscopic gastrostomy (PEG) tube feeding may be considered if dysphagia persists beyond the early subacute period in order to avoid long term complications of NG tube feeding.

c). Speech rehabilitation: Post stroke speech disorders which include aphasia and dysarthria occur in about $82.37 \%$ on admission (Vidović, Sinanović, Sabaskić, Haticić, \& Brkić, 2011). A significant number of them improve spontaneously with or without intervention. Treatment should be commenced early, as soon as the patient is able to participate. Speech-language pathologists play a significant role in the screening, formal assessment, management, and rehabilitation of stroke survivors who present with communication impairment. Speech-language therapy is grossly underdeveloped and even unavailable in most parts of the Sub-Saharan Africa (Wylie, McAllister, Davidson, \& Marshall, 2016).

d). Psychological rehabilitation: Post-stroke depression (PSD) is common and may occur in about $35 \%$ of stroke survivors (Srivastava, Taly, Gupta, \& Murali, 2010). The aetiology is multifactorial and includes the psychological effects of the disease, the effect of stroke on the brain, and the effects of some of the medications. Several studies suggested that large lesions in critical areas such as left frontal lobe and basal ganglia might interrupt the pathways of monoamines leading to depression (Chao, Min, \& Xue-Yuan, 2014). Psychotherapy is essential in the management of PSD and it is provided by the family members, clinical psychologist and the neurologist. Antidepressants are given to some patients as they also provide anxiolytic effects.

e). Occupational rehabilitation: Due to the neurological deficits associated with stroke, most patients may not return to their previous job. There may be a need to reassign duty in their previous job or change their occupation entirely to suit their current reality. This may involve training and retraining of the residual abilities to acquire new skills. This is done by occupational therapists.

Rehabilitation continues until the patient is adequately reintegrated back to the community. This is done concurrently with stroke prevention measures.

\subsection{Stroke Research}

Stroke research is essential pillar of stroke management. It involves the synthesis of empirical evidence from stroke prevention, acute stroke management, and stroke rehabilitation and application of the same in reduction of stroke morbidity and mortality. The level and strength of scientific evidence is derived from the scientific method applied in its synthesis. The applicable methods include expert opinions, observational studies, case-control studies, randomized controlled trials (RCTs), and meta-analysis in order of increasing strength. Stroke research should be done by all the health professionals involved in stroke management to help strengthen each pillar. The study can be done in the wards, outpatient clinics, laboratory, and the community. Most of the studies are sponsored by the researcher though can also be sponsored by the government, non-governmental organizations, and pharmaceutical companies. It is important to encourage RCTs in developing countries as there is danger of blanket application of research findings from developed nations due to genetic and environmental 
differences.

\subsection{Stroke Support}

This pillar plays a vital role in stroke management especially in the developing countries where health system is not well developed. Stroke occurs predominantly in low and middle income countries and its cost of management is very high (Owolabi et al., 2018; Kaur, Kwatra, Kaur, \& Pandian, 2014). There is also an absence and acute shortage of hyperacute stroke management services and investigative modalities like computed axial tomography (CT) scan, magnetic resonance imaging (MRI), and functional imaging modalities in most developing countries. This makes comprehensive stroke management services unavailable, unaffordable, and inaccessible to the populace, especially in the absence of universal health insurance coverage. There is a need for government, non-governmental organizations (NGOs) and philanthropists to step in to bridge the gap. There should be legislation on stroke care as provision of above facilities will significantly reduce stroke morbidity and mortality. This should include total or part sponsorship of stroke care, provision of 24 hour ambulance services to reduce the delays in patient hospital presentation, and also provision of all the investigative tools and treatment modalities in regional hubs of comprehensive stroke centres. Furthermore, NGOs and philanthropists could make contributions in reducing the infrastructural deficits, provision of psychosocial support to the stroke survivors, and support of stroke research.

\section{Conclusion}

The morbidity and mortality of stroke is very high especially in the developing countries due to poverty, ignorance, and unavailability of comprehensive stroke management services. There is a need to strengthen the available management steps as highlighted in the stroke pentagon and ensure that the stakeholders are responsive and responsible. This will help improve the availability, accessibility, affordability, and efficiency of stroke care and thereby reduce the burden of stroke.

\section{Competing Interests Statement}

The author declares that there are no competing or potential conflicts of interest.

\section{References}

Avan, A., Digaleh, H., Di Napoli, M., Stranges, S., Behrouz, R., Shojaeianbabaei, G., ... \& Amiri, A. (2019). Socioeconomic status and stroke incidence, prevalence, mortality, and worldwide burden: an ecological analysis from the Global Burden of Disease Study 2017. BMC Med, 17, 191. https://doi.org/10.1186/s12916-019-1397-3

Chao, F., Min, F., \& Xue-Yuan, L. (2014). The Neurobiological Pathogenesis of Poststroke Depression. The Scientific World Journal, 8, 2014. https://doi.org/10.1155/2014/521349

Eze, C. O., Agu, C. E., Kalu, U. A., Maduanusi, C. A., Nwali, S. T., \& Igwenyi, C. (2013). Pattern of Medical Admissions in a Tertiary Health Centre in Abakaliki South-East Nigeria. Journal of Biology, Agriculture and Healthcare, 3(12), 90-94.

Eze, C. O., \& Kalu, U. A. (2019). Pattern of Mortality in Medical Emergency Room: Experience at Abakaliki Nigeria. J Metabolic Synd, 8, 248.

Eze, C., Kalu, U., \& Isiguzo, G. (2019). Stroke in Young Adults: Experience at Abakaliki South East Nigeria. World Journal of Neuroscience, 9, 217-223. https://doi.org/10.4236/wjns.2019.94015

Eze, C., Kalu, U., \& Nnaji, T. (2020). Cardiovascular Risk Factors in South-Eastern Nigeria: A Community Based Survey. World Journal of Cardiovascular Diseases, 10(7), 417-424. https://doi.org/10.4236/wjcd.2020.107040

Eze, C., Okoro, F., Nnaji, T., Nwobodo, M., Kalu, U., \& Ewah, R. (2020). Mortality Pattern in Intensive Care Unit: Experience at AbakalikiSoutheastern Nigeria. World Journal of Cardiovascular Diseases, 10(7), 473-482. https://doi.org/10.4236/wjcd.2020.107047

Eze, C. O., Okoro, F. C., Nwobodo, M. U., Nnaji, T., \& Isiguzo, G. C. (2020). Pattern of Mortality in Medical Wards: Experience at Abakaliki Nigeria. Int Res $J$ of Med Biomed Sci, 5(4), 36-41. https://doi.org/10.15739/irjmbs.20.008

Ezeala-Adikaibe, B., Aneke, E., Orjioke, C., Ezeala-Adikaibe, N., Mbadiwe, N., Chime, P., \& Okafor, U. (2014). Pattern of medical admissions at Enugu State University of Science and Technology Teaching Hospital: a 5 year Review. Ann Med Health Sci Res, 4(3), 426-431. https://doi.org/10.4103/2141-9248.133472 
Ezejimofor, M. C., Uthman, O. A., Maduka, O., Ezeabasili, A. C., Onwuchekwa, A. C., Ezejimofor, B. C, ... \& Kandala, N. B. (2017). Stroke survivors in Nigeria: A door-to-door prevalence survey from the Niger Delta region. Journal of the Neurological Sciences, 372, 262-269. https://doi.org/10.1016/j.jns.2016.11.059

GBD 2015 Neurological Disorders Collaborator Group. (2017). Global, regional, and national burden of neurological disorders during 1990-2015: a systematic analysis for the Global Burden of Disease Study 2015. Lancet Neurol, 16, 877-97.

George, M. G., Tong, X., Kuklina, E. V., \& Labarthe, D. R. (2011). Trends in stroke hospitalizations and associated risk factors among children and young adults, 1995-2008. Ann Neurol, 70, 713-721. https://doi.org/10.1002/ana.22539

Harris, J. E., \& Eng, J. J. (2010). Strength training improves upper-limb function in individuals with stroke: a meta-analysis. Stroke, 41, 136-140. https://doi.org/10.1161/STROKEAHA.109.567438

Kaur, P., Kwatra, G., Kaur, R., \& Pandian, J. D. (2014). Cost of stroke in low and middle income countries: a systematic review. International Journal of Stroke, 9(6), 678- 682. https://doi.org/10.1111/ijs.12322

Khedr, E. M., Fawi, G., Abdela, M., Mohammed, T. A., Ahmed, M. A., \& El-Fetoh, N. A. (2014). Prevalence of ischemic and hemorrhagic strokes in Qena Governorate, Egypt: community-based study. Journal of Stroke and Cerebrovascular Diseases, 23, 1843-8. https://doi.org/10.1016/j.jstrokecerebrovasdis.2014.03.001

Kolb, B. (1995). Brain plasticity and behaviour. Mahwah, NJ: Lawrence Erlbaum.

Lackland, D. T., Roccella, E. J., Deutsch, A. F., Fornage, M., George, M. G., Howard, G., ... \& Towfighi, A. (2014). Factors influencing the decline in stroke mortality. Stroke, 45, 315-53. https://doi.org/10.1161/01.str.0000437068.30550.cf

Mann, G., Hankey, G. J., \& Cameron, D. (1999). Swallowing function after stroke: prognosis and prognostic factors at 6 months. Stroke, 30, 744-748. https://doi.org/10.1161/01.STR.30.4.744

Nedeltchev, K., der Maur, T. A., Georgiadis, D., Arnold, M., Caso, V., Mattle, H. P., .. \& Baumgartner, R. W. (2005). Ischaemic stroke in young adults: predictors of outcome and recurrence. J NeurolNeurosurg Psychiatry, 76(2), 191-195. https://doi.org/10.1136/jnnp.2004.040543

Owolabi, M. O., Sarfo, F., Akinyemi, R., Gebregziabher, M., Akpa, O., AkpaluA, ... \& Ovbiagele, B. (2018). SIREN Team; as part of H3Africa Consortium. Dominant modifiable risk factors for stroke in Ghana and Nigeria (SIREN): a case-control study. Lancet Glob Health, 6, 436-446. https://doi.org/10.1016/S2214-109X(18)30002-0

Pang, M. Y., Eng, J. J., Dawson, A. S., \& Gylfadottir, S. (2006). The use of aerobic exercise training in improving aerobic capacity in individuals with stroke: a meta-analysis. ClinRehabil, 20, 97-111. https://doi.org/10.1191/0269215506cr926oa

Romero, J. R., Morris, J., \& Pikula, A. (2008). Stroke prevention: modifying risk factors. TherAdvCardiovasc Dis, 2(4), 287-303. https://doi.org/10.1177/1753944708093847

Sacco, R. L., Kasner, S. E., Broderick, J. P., Caplan, L.R., Culebras, A., Elkind, M. S., ... \& Vinters, H. V. (2013). An Updated Definition of Stroke for the 21st Century. Stroke, 44, 2064-2089. https://doi.org/10.1161/STR.0b013e318296aeca

Smithard, D. G., O'Neill, P. A., Park, C., Morris, J., Wyatt, R., England, R., \& Martin, D. F. (1996). Complications and outcome after stroke: does dysphagia matter? Stroke, 27, 1200-1204. https://doi.org/10.1161/01.STR.27.7.1200

Srivastava, A., Taly, A. B., Gupta, A., \& Murali, T. (2010). Post-stroke depression: prevalence and relationship with disability in chronic stroke survivors. Ann Indian Acad Neurol, 13(2), 123-127. https://doi.org/10.4103/0972-2327.64643

Varona, J. F., Guerra, J. M., Bermejo, F., Molina, J. A., \& de la Camara, G. (2007). Causes of ischemic stroke in young adults, and evolution of the etiological diagnosis over the long term. Eur Neurol, 57, 212-218. https://doi.org/10.1159/000099161

Veerbeek, J. M., Koolstra, M., Ket, J. C., van Wegen, E. E., \& Kwakkel, G. (2011). Effects of augmented exercise therapy on outcome of gait and gait-related activities in the first 6 months after stroke: a meta-analysis. Stroke, 42, 3311-3315. https://doi.org/10.1161/STROKEAHA.111.623819

Vidović, M., Sinanović, O., Sabaskić, L., Haticić, A., \& Brkić, E. (2011). Incidence and types of speech 
disorders in stroke patients. Actaclinica Croatica, 50, 491-4.

Walker, R. W., Jusabani, A., Aris, E., Gray, W. K., Whiting, D., \& Kabadi, G. (2011).Post-stroke case fatality within an incident population in rural Tanzania. J NeurolNeurosurg Psychiatry, 82, 1001-1005. https://doi.org/10.1136/jnnp.2010.231944

World Health Organization [WHO]. (2013). Projections of mortality and causes of death, 2015 and 2030. Health statistics and information systems. Retrieved June 21, 2016, from http://www.who.int/entity/healthinfo/global_burden_disease/GHE_DthGlobal_Proj_2015_2030.xls?ua=1

Wylie, K., McAllister, L., Davidson, B., \& Marshall, J. (2016). Communication rehabilitation in sub-Saharan Africa: A workforce profile of speech and language therapists. African journal of disability, 5(1), 227. https://doi.org/10.4102/ajod.v5i1.227

\section{Copyrights}

Copyright for this article is retained by the author(s), with first publication rights granted to the journal.

This is an open-access article distributed under the terms and conditions of the Creative Commons Attribution license (http://creativecommons.org/licenses/by/4.0/). 